\title{
Mistake-Driven Mixture of Hierarchical Tag Context Trees
}

\author{
Masahiko Haruno \\ NTT Communication Science Laboratories \\ 1-1 Hikari-No-Oka Yokosuka-Shi \\ Kanagawa 239, Japan \\ haruno@cslab.kecl.ntt.co.jp
}

\author{
Yuji Matsumoto \\ NAIST \\ 8916-5 Takavama-cho Ikoma-Shi \\ Nara 630-01, Japan \\ matsu@is.aist-nara.ac.jp
}

\begin{abstract}
This paper proposes a mistake-driven mixture method for learning a tag model. The method iteratively performs two procedures: 1 . constructing a tag model based on the current data distribution and 2 . updating the distribution by focusing on data that are not well predicted by the constructed model. The final tag model is constructed by mixing all the models according to their performance. To well reflect the data distribution, we represent each tag model as a hierarchical tag (i.e., $\mathrm{NTT}^{1}<$ proper noun $<$ noun) context tree. By using the hierarchical tag context tree, the constituents of sequential tag models gradually change from broad coverage tags (e.g.,noun) to specific exceptional words that cannot be captured by general tags. In other words, the method incorporates not only frequent connections but also infrequent ones that are often considered to be collocational. We evaluate several tag models by implementing Japanese part-of-speech taggers that share all other conditions (i.e.,dictionary and word model) other than their tag models. The experimental results show the proposed method significantly outperforms both hand-crafted and conventional statistical methods.
\end{abstract}

\section{Introduction}

The last few years have seen the great success of stochastic part-of-speech (POS) taggers (Church, 1988; Kupiec, 1992; Charniak et al., 1993; Brill, 1992; Nagata, 1994). The stochastic approach generally attains 94 to $96 \%$ accuracy and replaces the labor-intensive compilation of linguistics rules by using an automated learning algorithm. However,

${ }^{1}$ NTT is an abbreviation of Nippon Telegraph and Telephone Corporation. practical systems require more accuracy because POS tagging is an inevitable pre-processing step for all practical systems.

To derive a new stochastic tagger, we have two options since stochastic taggers generally comprise two components: word model and tag model. The word model is a set of probabilities that a word occurs with a tag (part-of-speech) when given the preceding words and their tags in a sentence. On the contrary, the tag model is a set of probabilities that a tag appears after the preceding words and their tags.

The first option is to construct more sophisticated word models. (Charniak et al., 1993) reports that their model considers the roots and suffixes of words to greatly improve tagging accuracy for English corpora. However, the word model approach has the following shortcomings:

- For agglutinative languages such as Japanese and Chinese, the simple Bayes transfer rule is inapplicable because the word length of a sentence is not fixed in all possible segmentations ${ }^{2}$. We can only use simpler word models in these languages.

- Sophisticated word models largely depend on the target language. It is time-consuming to compile fine-grained word models for each language.

The second option is to devise a new tag model. (Schütze and Singer. 1994) have introduced a variable-memory-length tag model. Unlike conventional bi-gram and tri-gram models, the method selects the optimal length by using the context tree (Rissanen, 1983) which was originally introduced for use in data compression (Cover and Thomas, 1991). Although the variable-memory length approach remarkably reduces the number of parameters, tagging accuracy is only as good as conventional methods. Why didn't the method have higher accuracy? The crucial problem for current

\footnotetext{
${ }^{2}$ In $P\left(w_{i} \mid t_{i}\right)=\frac{P\left(w_{1}\right) P\left(i_{1} \mid w_{1}\right)}{P\left(t_{1}\right)}, P\left(w_{i}\right)$ cannot be considered to be identical for all segmentations.
} 
tag models is the set of collocational sequences of words that cannot be captured by just their tags. Because the maximal likelihood estimator (MLE) emphasizes the most frequent connections, an exceptional connection is placed in the same class as a frequent connection.

To tackle this problem, we introduce a new tag model based on the mistake-driven mixture of hierarchical tag context trees. Compared to Schütze and Singer's context tree (Schütze and Singer, 1994), the hierarchical tag context tree is extended in that the context is represented by a hierarchical tag set (i.e..NTT < proper noun < noun). This is extremely useful in capturing exceptional connections that can be detected only at the word level.

To make the best use of the hierarchical context tree, the mistake-driven mixture method imitates the process in which linguists incorporate exceptional connections into hand-crafted rules: They first construct coarse rules which seems to cover broad range of data. They then try to analyze data by using the rules and extract exceptions that the rules cannot handle. Next they generalize the exceptions and refine the previous rules. The following two steps abstract the human algorithm for incorporating exceptional connections.

1. construct temporary rules which seem to well generalize given data.

2 . try to analyze data by using the constructed rules and extract the exceptions that cannot be correctly handled, then return to the first step and focus on the exceptions.

To put the above idea into our learning algorithm, The mistake-driven mixture method attaches a weight vector to each example and iteratively performs the following two procedures in the training phase:

1. constructing a context tree based on the current data distribution (weight vector)

2. updating the distribution (weight vector) by focusing on data not well predicted by the constructed tree. More precisely, the algorithm reduces the weight of examples that are correctly handled.

For the prediction phase, it then outputs a final tag model by mixing all the constructed models according to their performance. By using the hierarchical tag context tree, the constituents of a series of tag models gradually change from broad coverage tags (e.g.,noun) to specific exceptional words that cannot be captured by general tags. In other words, the method incorporates not only frequent connections but also infrequent ones that are often considered to be exceptional.

The construction of the paper is as follows. Section 2 describes the stochastic POS tagging scheme and hierarchical tag setting. Section 3 presents a new probability estimator that uses a hierarchical tag context tree and Section 4 explains the mistakedriven mixture method. Section 5 reports a preliminary evaluation using Japanese newspaper articles. We tested several tag models by keeping all other conditions (i.e., dictionary and word model) identical. The experimental results show that the proposed method significantly outperforms both handcrafted and conventional statistical methods. Section 6 concerns related works and Sections 7 concludes the paper.

\section{Preliminaries}

\subsection{Basic Equation}

In this section, we will briefly review the basic equations for part-of-speech tagging and introduce hierarchical-tag setting.

The tagging problem is formally defined as finding a sequence of tags $t_{1, n}$ that maximize the probability of input string $L$.

$$
\begin{array}{r}
\operatorname{argmax}_{t_{1, n}} P\left(w_{1, n}, t_{1, n} \mid L\right)=\operatorname{argmax}_{t_{1, n}} \frac{P\left(w_{1, n}, t_{1, n}, L\right)}{P(L)} \\
\Leftrightarrow \operatorname{argmax}_{t_{1, n}, w_{1, n} \in L} P\left(t_{1, n}, w_{1, n}\right)
\end{array}
$$

We break out $P\left(t_{1, n}, w_{1, n}\right)$ as a sequence of the products of tag probability and word probability.

$$
P\left(t_{1, n}, w_{1, n}\right)=\prod_{i=1}^{n} P\left(w_{i} \mid t_{1, i-1}, w_{1, i-1}\right) P\left(t_{i} \mid t_{1, i-1}, w_{1, i}\right)
$$

By approximating word probability as constrained only by its tag, we obtain equation (1). Equation (1) yields various types of stochastic taggers. For example, bi-gram and tri-gram models approximate their tag probability as $P\left(t_{i} \mid t_{i-1}\right)$ and $P\left(t_{i} \mid t_{i-1}, t_{i-2}\right)$, respectively. In the rest of the paper, we assume all tagging methods share the word model $P\left(w_{i} \mid t_{i}\right)$ and differ only in the tag model $P\left(t_{i} \mid t_{1, i-1}, w_{1, i}\right)$.

$\operatorname{argmax}_{t_{1, n}, w_{1, n} \in L} \prod_{i=1}^{n} P\left(t_{i} \mid t_{1, i-1}, w_{1, i}\right) P\left(w_{i} \mid t_{i}\right)$

\subsection{Hierarchical Tag Set}

To construct a tag model that captures exceptional connections, we have to consider word-level context as well as tag-level. In a more general form, we introduce a tag set that has a hierarchical structure. Our tag set has a three-level structure as shown in Figure 1. The topmost and the second level of the hierarchy are part-of-speech level and part-of-speech subdivision level respectively. Although stochastic taggers usually make use of subdivision level, part-of-speech level is remarkably robust 


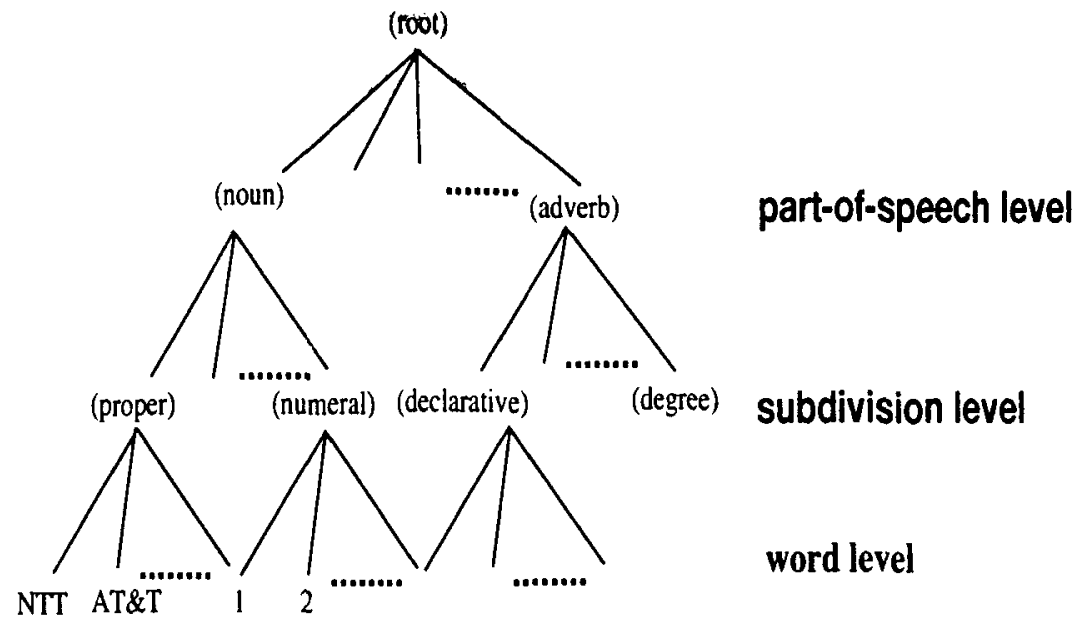

Figure 1: Hierarchical Tag Set

against data sparseness. The bottom level is word level and is indispensable in coping with exceptional and collocational sequences of words. Our objective is to construct a tag model that precisely evaluates $P\left(t_{i} \mid t_{1, i-1}, w_{1, i}\right)$ (in equation (1)) by using the threelevel tag set.

To construct this model, we have to answer the following questions.

1. Which level is appropriate for $t_{i}$ ?

2. Which length is to be considered for $t_{1, i-1}$ and $w_{1, i}$ ?

3. Which level is appropriate for $t_{1, i-1}$ and $w_{1, i}$ ?

To resolve the first question, we fix $t_{i}$ at subdivision level as is done in other tag models. The second and third questions are resolved by introducing hierarchical tag context trees and mistake-driven mixture method that are respectively described in Section 3 and 4 .

Before moving to the next section, let us define the basic tag set. If all words are considered context candidates, the search space will be enormous. Thus, it is reasonable for the tagger to constrain the candidates to frequent open class words and closed class words. The basic tag set is a set of the most detailed context elements that comprises the words selected above and part-of-speech subdivision level.

\section{Hierarchical Tag Context Tree}

A hierarchical tag context tree is constructed by a two-step methodology. The first step produces a context tree by using the basic tag set. The second step then produces the hierarchical tag context tree. It generalizes the basic tag context tree and avoids over-fitting the data by replacing excessively specific context in the tree with more general tags.
Finally, the generated tree is transformed into a finite automaton to improve tagging efficiency (Ron et al., 1997).

\subsection{Constructing a Basic Tag Context Tree}

In this section, we construct a basic tag context tree. Before going into detail of the algorithm, we briefly explain the context tree by using a simple binary case. The context tree was originally introduced in the field of data compression (Rissanen, 1983; Willems et al., 1995; Cover and Thomas, 1991) to represent how many times and in what context each symbol appeared in a sequence of symbols. Figure 2 exemplifies two context trees comprising binary symbols ' $a$ ' and ' $b$ '. $T(4)$ is constructed from the sequence ' $b a a b$ ' and $\mathrm{T}(6)$ from 'baabab'. The root node of $T(4)$ explains that both ' $a$ ' and ' $b$ ' appeared twice in 'baab' when no consideration is taken of previous symbols. The nodes of depth 1 represent an order 1 (bi-gram) model. The left node of $\mathrm{T}(4)$ represents that both ' $a$ ' and ' $b$ ' appeared only once after symbol ' $a$ ', while the right node of $T(4)$ represents only ' $a$ ' occurred once after ' $b$ '. In the same way, the node of depth 2 in $T(6)$ represents an order 2 (tri-gram) context model.

It is straightforward to extend this binary tree to a basic tag context tree. In this case, context symbols ${ }^{\prime} a$ and ${ }^{\prime} b{ }^{\prime}$ are replaced by an element of the basic tag set and the frequency table of each node then consists of the part-of-speech subdivision set.

The procedure construct-btree which constructs a basic tag context tree is given below. Let a set of subdivision tags to be $s_{1}, \cdots, s_{n}$. Let weight $[t]$ be a weight vector attached to the $t$ th example $x(t)$. Initial values of weight $[t]$ are set to 1 .

1. the only node, the root, is marked with the count table $\left(c\left(s_{1}, \lambda\right), \cdots, c\left(s_{n}, \lambda\right)=(0, \cdots, 0)\right)$.

2. Apply the following recursively. Let $T(t-1)$ be 


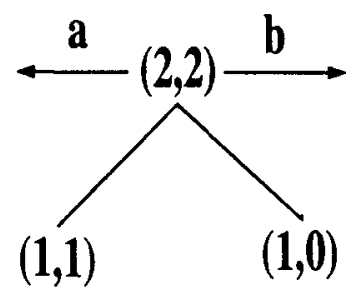

$T(4)$

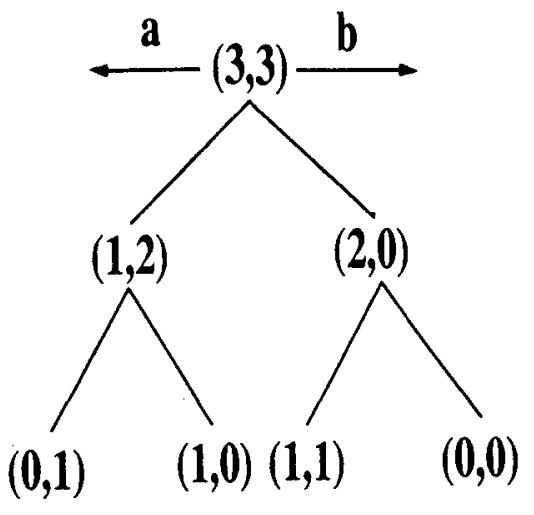

$T(6)$

Figure 2: Context Trees for ' $b a a b^{\prime}$ and ${ }^{\prime} b a a b a b{ }^{\prime}$

the last constructed tree with counts of nodes $z,\left(c\left(s_{1}, z\right), \cdots, c\left(s_{n}, z\right)\right)$. After the next symbol whose subdivision is $x(t)$ is observed, generate the next tree $T(t)$ as follows: follow the $T(t-1)$, starting at the root and taking the branch indicated by each successive symbol in the past sequence by using basic tag level. For each node $z$ visited, increment the component count $\mathrm{c}(x(t), z)$ by weight $[t]$. Continue until node $w$ is a leaf node.

3 . If $w$ is a leaf, extend the tree by creating new leaves: $c\left(x(t), w s_{1}\right)=\cdots=\mathrm{c}\left(x(t), w s_{n}\right)$ $=$ weight $[t], \mathrm{c}\left(\overline{x(t)}, w s_{1}\right)=\cdots=\mathrm{c}\left(\overline{x(t)}, w s_{n}\right)=0$. Define the resulting tree to be $T(t)$.

\subsection{Constructing a Hierarchical Tag Context Tree}

This section delineates how a hierarchical tag context tree is constructed from a basic tag context tree. Before describing the algorithm, we prepare some definitions and notations.

Let $A$ be a part-of-speech subdivision set. As described in the previous section, frequency tables of each node consist of the set $A$. At any node $s$ of a context tree, let $n(a \mid s)$ and $\hat{P}(a \mid s)$ be the count of element $a$ and its probability, respectively.

$$
\dot{P}(a \mid s)=\frac{n(a \mid s)}{\sum_{b \subseteq .4} n(b \mid s)}
$$

We introduce an information-theoretical criteria $\Delta(s b)$ (Weinberger et al., 1995) to evaluate the gain of expanding a node $s$ by its daughter $s b$.

$$
\Delta(s b)=\sum_{a \subseteq A} n(a \mid s b) \log \frac{\dot{P}(a \mid s b)}{\dot{P}(a \mid s)}
$$

$\Delta(s b)$ is the difference in optimal code lengths when symbols at node $s b$ are compressed by using probability distribution $\dot{P}(\cdot \mid s)$ at node $s$ and $\dot{P}(\cdot \mid s b)$ at node $s b$. Thus, the larger $\Delta(s b)$ is, the more meaningful it is to expand a node by $s b$.

Now, we go back to the hierarchical tag context tree construction. As illustrated in Figure 3, the generation process amounts to the iterative selection of $b$ out of word level, subdivision, part-of-speech and null (no expansion). Let us look at the procedure from the information-theoretical viewpoint. Breaking out equation (2) as $(3), \Delta(s b)$ is represented as the product of the frequencies of all subdivision symbols at node $s b$ and Kullback-Leibler (KL) divergence.

$$
\begin{aligned}
\Delta(s b) & =n(s b) \sum_{a \subseteq A} \frac{n(a \mid s b)}{n(s b)} \log \frac{\hat{P}(a \mid s b)}{\hat{P}(a \mid s)} \\
& =n(s b) \sum_{a \subseteq A} \dot{P}(a \mid s b) \log \frac{\dot{P}(a \mid s b)}{\dot{P}(a \mid s)} \\
& =n(s b) D_{K L}(\dot{P}(\cdot \mid s b), \dot{P}(\cdot \mid s))
\end{aligned}
$$

Because the $\mathrm{KL}$ divergence defines a distance measure between probability distributions, $\dot{P}(\cdot \mid s b)$ and $\dot{P}(\cdot \mid s)$, there is the following trade-off between the two terms of equation (3).

- The more general $b$ is, the more subdivision symbols appear at node $s b$.

- The more specific $b$ is, the more $\dot{P}(\cdot \mid s)$ and $\dot{P}(\cdot \mid s b)$ differ.

$\mathrm{By}$ using the trade-off, the optimal level of $b$ is selected.

Table 1 summarizes the algorithm construct-htree that constructs the hierarchical tag context tree. First, construct-htree generates a basic tag context tree by calling construct-btree. Assume that the 


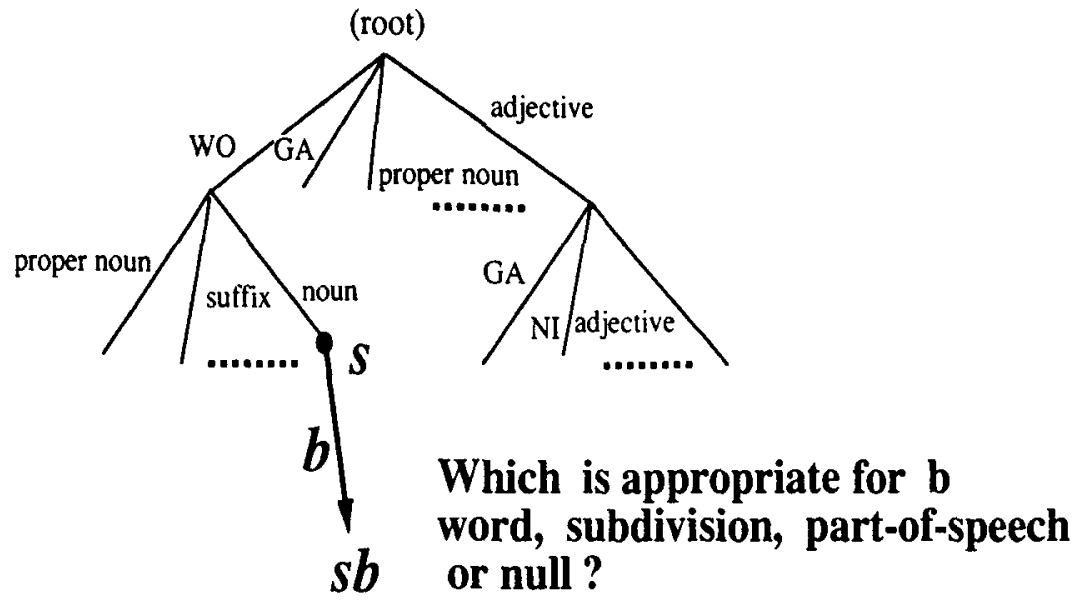

Figure 3: Constructing Hierarchical Tag Context Tree

training examples consist of a sequence of triples, $<p_{t}, s_{t}, w_{t}>$, in which $p_{t}, s_{t}$ and $w_{t}$ represent part-of-speech, subdivision and word, respectively. Eachtime the algorithm reads an example, it first reaches current leaf node $s$ by following the past sequence, computes $\Delta(s b)$, and then selects the optimal $b$. The initially constructed basic tag context tree is used to compute $\Delta(s b)$ s.

\section{Mistake-Driven Mixture of Hierarchical Tag Context Trees}

Up to this section, we introduced a new tag model that uses a single hierarchical tag context tree to cope with the exceptional connections that cannot be captured by just part-of-speech level. However, this approach has a clear limitation; the exceptional connections that do not occur so often cannot be detected by the single tree model. In such a case, the first term $n(s b)$ in equation (3) is enormous for general $b$ and the tree is expanded by using more general symbols.

To overcome this limitation, we devised the mistake-driven mixture algorithm summarized in $\mathrm{Ta}$ ble 4 which constructs $T$ context trees and outputs the final tag model.

mistake-driven mixture sets the weights to 1 for all examples and repeats the following procedures $T$ times. The algorithm first construct a hierarchical context tree by using the current weight vector. Example data are then tagged by the tree and the weights of correctly handled examples are reduced by equation (4). Finally, the final tag model is constructed by mixing $T$ trees according to equation (5).

By using the mistake-driven mixture method, the constituents of a series of hierarchical tag context trees gradually change from broad coverage tags (e.g.,noun) to specific exceptional words that cannot be captured by part-of-speech and subdivisions. The method, by mixing different levels of trees, incorporates not only frequent connections but also infrequent ones that are often considered to be collocational without over-fitting the data.

\section{Preliminary Evaluation}

We performed an preliminary evaluation using the first 8939 Japanese sentences in a year's volume of newspaper articles(Mainichi, 1993). We first auto matically segmented and tagged these sentences and then revised them by hand. The total number of words in the hand-revised corpus was 226162 . We trained our tag models on the corpora with every tenth sentence removed (starting with the first sentence) and then tested the removed sentences. There were 22937 words in the test corpus.

As the first milestone of performance, we tested a hand-crafted tag model of JUMAN (Kurohashi et al., 1994), the most widely used Japanese part-ofspeech tagger. The tagging accuracy of JUMAN for the test corpus was only $92.0 \%$. This shows that our corpus is difficult to tag because the corpus contains various genres of texts; from obituaries to poetry.

Next, we compared the mixture of bi-grams and the mixture of hierarchical tag context trees. In this experiment, only post-positional particles and auxiliaries were word-level elements of basic tags and all other elements were subdivision level. In contrast, bi-gram was constructed by using subdivision level. We set the iteration number $T$ to 5 . The results of our experiments are summarized in Figure 4.

As a single tree estimator (Number of Mixture = 1), the hierarchical tag context tree attained $94.1 \%$ accuracy, while bi-gram yielded $93.1 \%$. A hierarchical tag context tree offers a slight improvement, but 


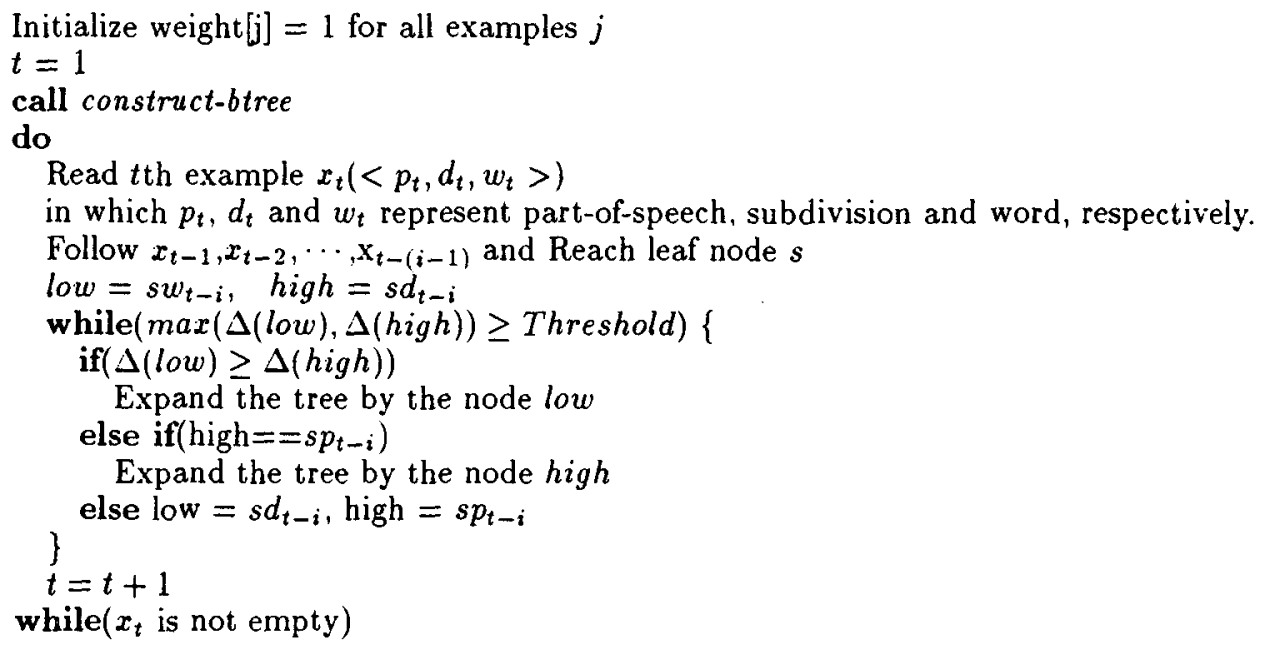

Table 1: Algorithm construct-htree

Input: sequence of $V$ examples $\left\langle p_{1}, d_{1}, w_{1}\right\rangle, \ldots,\left\langle p_{N}, d_{N}, w_{N}\right\rangle$

in which $p_{i}, d_{i}$ and $w_{i}$ represent part-of-speech, subdivision and word, respectively.

Initialize the weight vector weight $[i]=1$ for $i=1, \ldots, N$

Do for $t=1,2, \ldots, T$

Call construct-htree providing it with the weight vector weight $\square$ and

Construct a part-of-speech tagger $h_{t}$

Let Error be a set of examples that are not identified by $h_{t}$

Compute the error rate of $h_{t}: \epsilon_{t}=\sum_{i \subset E \text { rror }}$ weight $[i] / \sum_{i=1}^{N}$ weight $[i]$

$\beta_{t}=\frac{\epsilon_{t}}{1-\epsilon_{t}}$

For examples correctly predicted by $h_{t}$, update the weights vector to be weight $[\mathrm{i}]=$ weight $[\mathrm{i}] \beta_{t}$

Output a final tag model

$$
h_{f}=\sum_{t=1}^{T}\left(\log \frac{1}{\beta_{t}}\right) h_{t} / \sum_{t=1}^{T}\left(\log \frac{1}{\beta_{t}}\right)
$$

Table 2: Algorithm mistake-driven mixture

not a gret deal. This conclusion agrees with Schütze and Singer's experiments that used a context tree of usual part-of-speech.

When we turn to the mixture estimator, a great difference is seen between hierarchical tag context trees and bi-grams. The hierarchical tag context trees produced by the mistake-driven mixture method, greatly improved the accuracy and overfitting data was not serious. The best and worst performances were $96.1 \%$ (Number of Mixture $=3$ ) and $94.1 \%$ (Number of Mixture $=1$ ), respectively. On the other hand, the performance of the bi-gram mixture was not satisfactory. The best and worst performances were $93.8 \%$ (Number of Mixture $=2$ ) and $90.8 \%$ (Number of Mixture $=5$ ), respectively.

From the result, we may say exceptional connections are well captured by hierarchical context trees but not by bi-grams. Bi-grams of subdivision are too general to selectively detect exceptions.

\section{Related Work}

Although statistical natural language processing has mainly focused on Maximum Likelihood Estimators, (Pereira et al., 1995) proposed a mixture approach to predict next words by using the Context Tree Weighting (CTW) method (Willems et al., 1995). The CTW method computes probability by mixing subtrees in a single context tree in Bayesian fashion. Although the method is very efficient, it cannot be used to construct hierarchical tag context trees.

Various kinds of re-sampling techniques have been studied in statistics (Efron, 1979; Efron and Tibshirani, 1993) and machine learning (Breiman, 1996; Hull et al., 1996; Freund and Schapire, 1996a). In particular, the mistake-driven mixture algorithm 


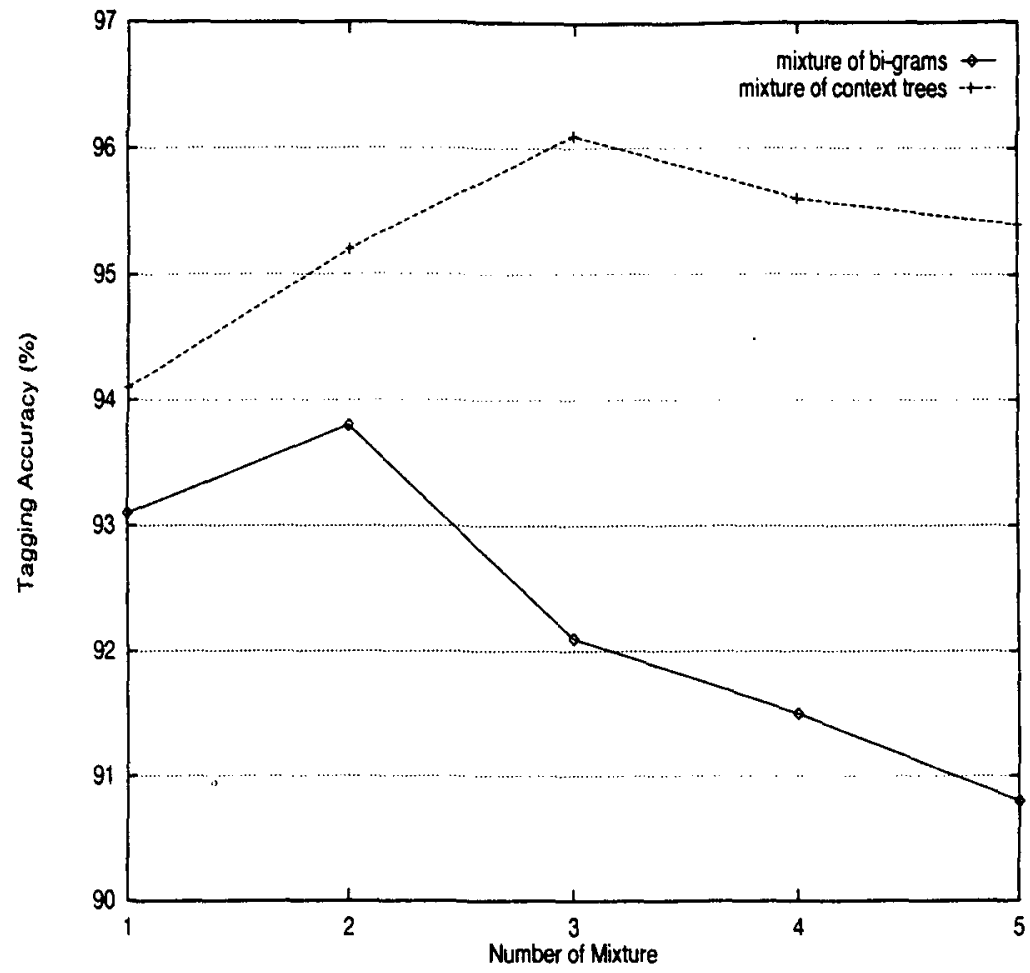

Figure 4: Context Tree Mixture v.s. Bi-gram Mixture

was directly motivated by Adaboost (Freund and Schapire, 1996a). The Adaboost method was designed to construct a high-performance predictor by iteratively calling a weak learning algorithm (that is slightly better than random guess). An empirical work reports that the method greatly improved the performance of decision-tree, $k$-nearestneighbor, and other learning methods given relatively simple and sparse data (Freund and Schapire, 1996b). We borrowed the idea of re-sampling to detect exceptional connections and first proved that such a re-sampling method is also effective for a practical application using a large amount of data. The next step is to fill the gap between theory and practition. Most theoretical work on re-sampling assumes i.i.d (identically, independently distributed) samples. This is not a realistic assumption in partof-speech tagging and other NL applications. An interesting future research direction is to construct a theory that handles Markov processes.

\section{Conclusion}

We have described a new tag model that uses mistake-driven mixture to produce hierarchical tag context trees that can deal with exceptional connections whose detection is not possible at part-ofspeech level. Our experimental results show that combining hierarchical tag context trees with the mistake-driven mixture method is extremely effective for 1 . incorporating exceptional connections and 2. avoiding data over-fitting. Although we have focused on part-of-speech tagging in this paper, the mistake-driven mixture method should be useful for other applications because detecting and incorporating exceptions is a central problem in corpus-based NLP. We are now costructing a Japanese dependency parser that employes mistake-driven mixture of decision trees.

\section{References}

Leo Breiman. 1996. Bagging predictors, Machine Learning, 24(2):123-140, August.

Eric Brill. 1992. A simple rule-based part of speech tagger. In Proc. Third Conference on Applied Natural Language Processing, pages 152-155.

Eugene Charniak, Curtis Hendrickson, Neil Jacobson, and Mike Perkowits. 1993. Equations for Part-of-Speech Tagging. In Proc. 11th AAAI, pages $784-i 89$.

K. W. Church. 1988. A stochastic parts program and noun phrase parser for unrestricted text. In Proc. ACL 2nd Conference on Applied Natural Language Processing, pages 126-143. 
T.M. Cover and J.A. Thomas, 1991. Elements of Information Theory. John Wiley \& Sons.

B. Efron and R. Tibshirani, 1993. An Introduction to the Bootstrap. Chapman and Hall.

B. Efron. 1979. Bootstrap: another look at the jackknife. The Annals of Statistics, 7(1):1-26.

Yoav Freund and Robert Schapire. 1996a. A decision-theoretic generalization of on-line learning and an application to boosting.

Yoav Freund and Robert Schapire. 1996b. Experiments with a New Boosting algorithm. In Proc. 13rd International Conference on Machine Learning, pages 148-156.

David A. Hull, Jan O. Pedersen, and Hinrich Schütze. 1996. Method combination for document filtering. In Proc. ACM SIGIR 96, pages 279-287.

J. Kupiec. 1992. Robust part-of-speech tagging using a hidden Markov model. Computer Speech and Language, 6:225-242.

Sadao Kurohashi, Toshihisa Nakamura, Yuji Matsumoto, and Makoto Nagao. 1994. Improvements of Japanese morphological analyzer juman. In Proc. International Workshop on Sharable Natural Language Resources, pages 22-28.

Mainichi, 1993. CD Mainichi Shinbun. Nichigai Associates Co.

Masaaki Nagata. 1994. A Stochastic Japanese Morphological Analyzer Using Forward-DP Backward- $A^{*}$ N-Best Search Algorithm. In Proc. 15th COLING, pages 201-207.

Fernando C. Pereira, Yoram Singer, and Naftali Tishby. 1995. Beyond Word N-Grams. In Proc. Third Workshop on Very Large Corpora, pages 95-106.

Jorma Rissanen. 1983. A universal data compression system. IEEE Transaction on Information Theory, 29(5):656-664, September.

Dana Ron, Yoram Singer, and Naftali Tishby. 1997. The power of amnesia: Learning probabilistic automata with variable memory length. (to appear) Machine Learning Special Issue on COLT94.

H. Schütze and Y. Singer. 1994. Part-of-speech tagging using a variable markov model. In the $32 \mathrm{th}$ Annual Meeting of ACL, pages 181-18T.

M J. Weinberger, J J. Rissanen, and M. Feder. 1995. A universal finite memory source. IEEE Transaction on Information Theory, 41(3):643-652, May.

F M J. Willems, Y M. Shtarkov, and T J. Tjalkens. 1995. The context-tree weigting method: Basic properties. IEEE Transaction on Information Theory, 41(3):653-664, May. 